\title{
Tobamoviral Movement Protein Transiently Expressed in a Single Epidermal Cell Functions Beyond Multiple Plasmodesmata and Spreads Multicellularly in an Infection-Coupled Manner
}

\author{
Atsushi Tamai and Tetsuo Meshi \\ Department of Botany, Graduate School of Science, Kyoto University, Sakyo-ku, Kyoto 606-8502, Japan \\ Accepted 6 October 2000.
}

\begin{abstract}
Cell-to-cell movement of a plant virus requires expression of the movement protein (MP). It has not been fully elucidated, however, how the MP functions in primary infected cells. With the use of a microprojectile bombardmentmediated DNA infection system for Tomato mosaic virus (ToMV), we found that the cotransfected ToMV MP gene exerts its effects in the initially infected cells and in their surrounding cells to achieve multicellular spread of movement-defective ToMV. Five other tobamoviral MPs examined also transcomplemented the movementdefective phenotype of ToMV, but the Cucumber mosaic virus 3a MP did not. Together with the cell-to-cell movement of the mutant virus, a fusion between the MP and an enhanced green fluorescent protein variant (EGFP) expressed in trans was distributed multicellularly and localized primarily in plasmodesmata between infected cells. In contrast, in noninfected sites the MP-EGFP fusion accumulated predominantly inside the bombarded cells as irregularly shaped aggregates, and only a minute amount of the fusion was found in plasmodesmata. Thus, the behavior of ToMV MP is greatly modulated in the presence of a replicating virus and it is highly likely that the MP spreads in the infection sites, coordinating with the cell-to-cell movement of the viral genome.
\end{abstract}

Systemic infection of a plant virus involves replication in initially infected cells, subsequent local cell-to-cell movement through plasmodesmata (the intercellular channels providing symplasmic continuity of plant cells) (Ding 1998; Lucas et al. 1993), and long-distance movement through the vascular tissues. It is well established that most plant viruses encode one or more proteins, referred to as movement proteins (MPs), required for their own cell-to-cell movement (Atabekov and Taliansky 1990; Deom et al. 1992; Maule 1991).

Tobacco mosaic virus (TMV) and related tobamoviruses encode a single 30-kDa MP (Deom et al. 1987; Meshi et al. 1987). TMV MP is localized in plasmodesmata of infected tobacco leaves (Tomenius et al. 1987) and in noninfected transgenic tobacco (Atkins et al. 1991; Ding et al. 1992) and is

Corresponding author: T. Meshi; Fax: +81-75-753-4141;

E-mail: tmeshi@gr.bot.kyoto-u.ac.jp thought to increase the size exclusion limit (SEL) of the plasmodesmal pores (Waigmann et al. 1994; Wolf et al. 1989; Wolf et al. 1991). At mid-to-late stages of infection, MP formed irregularly shaped aggregates inside the infected cells (Kawakami and Watanabe 1997; Meshi et al. 1992; Padgett et al. 1996) that involved the endoplasmic reticulum (ER) (Reichel and Beachy 1998) and later associated with microtubules and microfilaments (Heinlein et al. 1995; McLean et al. 1995). MP colocalized, albeit not completely, with plus- and minus-sense viral RNAs and a virus-encoded replicase component (Heinlein et al. 1998; Más and Beachy 1999). MP can bind to single-strand nucleic acids in vitro to form a filamentous ribonucleoprotein complex (Citovsky et al. 1990; Citovsky et al. 1992) and has an affinity for tubulin and actin (McLean et al. 1995). In light of these observations, it was proposed that the genomic RNA and MP assemble to form a viral ribonucleoprotein (vRNP) complex, which is then transported to plasmodesmata by the cytoskeletal network. Subsequently, the MP somehow modulates the plasmodesmal permeability, enabling vRNP to move through to the neighboring cells (Carrington et al. 1996; Citovsky 1999; Lazarowitz and Beachy 1999). Despite such vivid modeling of cell-to-cell movement of tobamovirus, the molecular mechanisms are still largely unknown and some observations appear to contradict each other.

When bacterially expressed MP was microinjected into mesophyll cells of tobacco, the plasmodesmal SEL rapidly (3 to $5 \mathrm{~min}$ ) increased from $<1$ to $>20 \mathrm{kDa}$ in both the injected and far-distant cells (Waigmann et al. 1994). The injected MP moved between the trichome cells of Nicotiana clevelandii without markedly increasing the basal plasmodesmal SEL of approximately $7 \mathrm{kDa}$ (Waigmann and Zambryski 1995). In the epidermal cells of TMV-infected $N$. benthamiana, virally expressed MP-green fluorescent protein (GFP) fusion was found only in and between infected cells and the increased plasmodesmal SEL occurred between cells inside the infection foci but not between the most recently infected cells and as yet uninfected immediate neighbors (Oparka et al. 1997). Moreover, a comparative study of two microinjection methods suggested that the increase in the plasmodesmal SEL in MPexpressing tobacco tissues might not reflect a genuine biochemical activity of the MP (Storms et al. 1998).

The function of MP during the infection process has, so far, been analyzed mainly with infectious transcripts synthesized 
in vitro and transgenic plants. These methods, however, result in expression of wild-type or modified MP in at least most of the infected cells and, therefore, the activity of MP in the primary infected cells may not have been fully delineated. Previously, Morozov et al. (1997) showed that a Potato virus X mutant defective in cell-to-cell movement spread when an infectious DNA producing the mutant virus was cobombarded with a separately cloned MP gene. This system makes it possible to introduce an MP gene in the primary infected cells and only to express it independently of virus infection. Here we report data from such transcomplementation experiments with infectious DNAs for GFP-tagged Tomato mosaic virus (ToMV) mutants. Results showed that tobamoviral MPs expressed in an epidermal cell enable movement-defective ToMV to spread multicellularly and infection greatly modulates the intercellular and intracellular distribution patterns of MP, implying a coordination of cell-to-cell spread and intracellular multiplication in tobamovirus infection.

\section{RESULTS}

DNA-mediated infection of ToMV.

We constructed a series of infectious plasmids from which replication-competent ToMV (L strain) derivatives were generated under the control of the Cauliflower mosaic virus (CaMV) 35S RNA promoter after transfection by micropro-

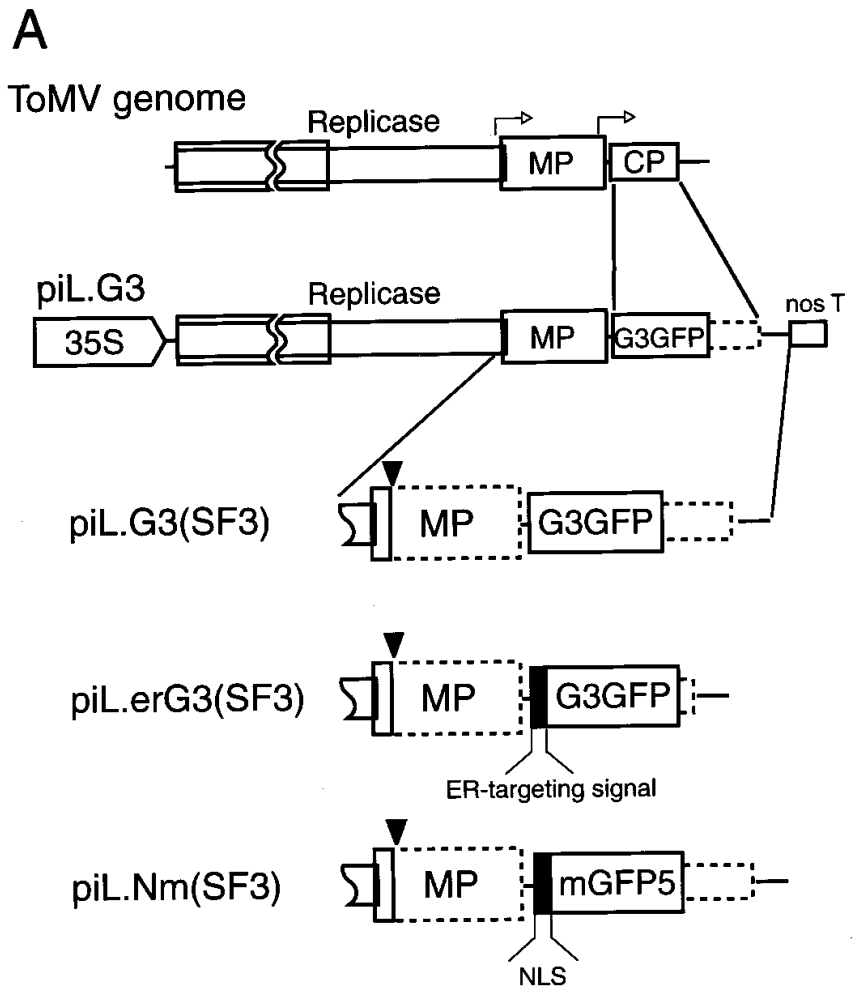

jectile bombardment. To monitor the infection, the coat protein (CP) gene, which is dispensable for replication and cellto-cell movement (Meshi et al. 1987; Takamatsu et al. 1987), was replaced with a GFP gene. The structures of infectious plasmids are illustrated in Fig. 1A and the characteristics of GFP variants are summarized in Table 1.

When piL.G3 encoding G3GFP (Kawakami and Watanabe 1997) as a reporter (Fig. 1A) was bombarded into the epidermal cells of nearly expanded leaves of $N$. benthamiana, clusters of GFP-expressing cells were observed 1 to 2 days after bombardment by fluorescent microscopy (Fig. 2A). No GFP signals were detected when a plasmid harboring a mutation

Table 1. Characteristics of green fluorescent protein (GFP) variants

\begin{tabular}{lccll}
\hline & \multicolumn{2}{c}{ Excitability } & \\
\cline { 2 - 3 } GFP variant $^{\mathbf{a}}$ & UV & Blue light & Subcellular localization \\
\hline G3GFP $(\mathrm{G} 3)$ & - & + & Nucleus $\sim$ cytosol \\
NmGFP $(\mathrm{Nm})$ & + & + & Nucleus $>$ cytosol \\
erG3GFP $(\mathrm{erG3})$ & - & + & Endoplasmic reticulum $(\mathrm{ER})$ \\
mGFP5ER $_{\text {EGFPb }}$ & + & + & ER \\
ER & - & + & Nucleus $\sim$ cytosol \\
\hline
\end{tabular}

a Abbreviations used in the plasmid names are shown in parentheses.

${ }^{b}$ An enhanced green fluorescent protein variant (EGFP) was used only as the fusion protein with Tomato mosaic virus movement protein. Sequence of the EGFP gene differs considerably (approximately $77 \%$ identity) from the coding sequences for the other GFP variants listed.

B

p35LM (ToMV MP)

p35LME (MP-EGFP)

pBIG3 (G3GFP)<smiles>[B][Ba][Ba][Ba][Ba]</smiles>

(erG3GFP)

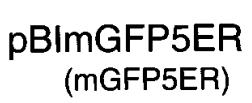

$\mathrm{pBINm}$

(NmGFP)
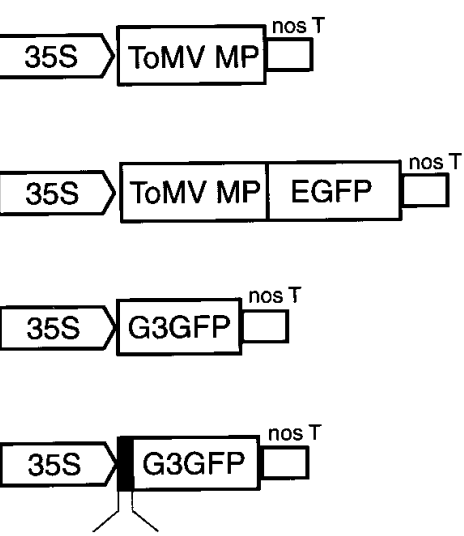

ER-targeting signal
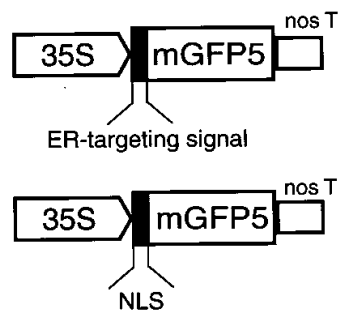

Fig. 1. A, Schematic representation of infectious Tomato mosaic virus (ToMV) plasmids. Genomic organization of ToMV is shown at the top. Boxes = coding regions. Thin lines $=$ noncoding regions. Bent arrows = starting positions of the subgenomic mRNAs. In piL.G3, the pentagon incidicates the Cauliflower mosaic virus 35S RNA promoter (35S) and the small box indicates the nopaline synthase gene terminator (nosT). Boxes delineated with broken lines = nonexpressed portions derived from the movement protein (MP) or coat protein gene. The portions corresponding to the endoplasmic reticulum-targeting signal and nuclear localization signal are in black. Closed triangles $=$ SF3 frame-shift mutation introduced at the tenth codon of the MP gene. B, Schematic representation of the genes for ToMV MP, ToMV MP-green fluorescent protein variant, and green fluorescent protein derivatives, which are expressed under the control of the $35 \mathrm{~S}$ promoter. The protein expressed from each plasmid is shown in parentheses below the name of the plasmid. 
in the replicase gene was bombarded (data not shown). Therefore, the GFP signals observed were not derived from any mRNA mRNA transcribed directly from the bombarded plasmids but from the subgenomic mRNA produced during the course of viral multiplication. When the MP gene of the infectious plasmid was mutated, the GFP signals were restricted to single cells (Fig. 2B) for a frame-shift mutant derived from piL.G3(SF3) (Fig. 1A). The same result occurred with MP mutants that had a point mutation, resulting in an amino acid change from Ser37 to Ala (Kawakami et al. 1999) or an internal deletion (data not shown).
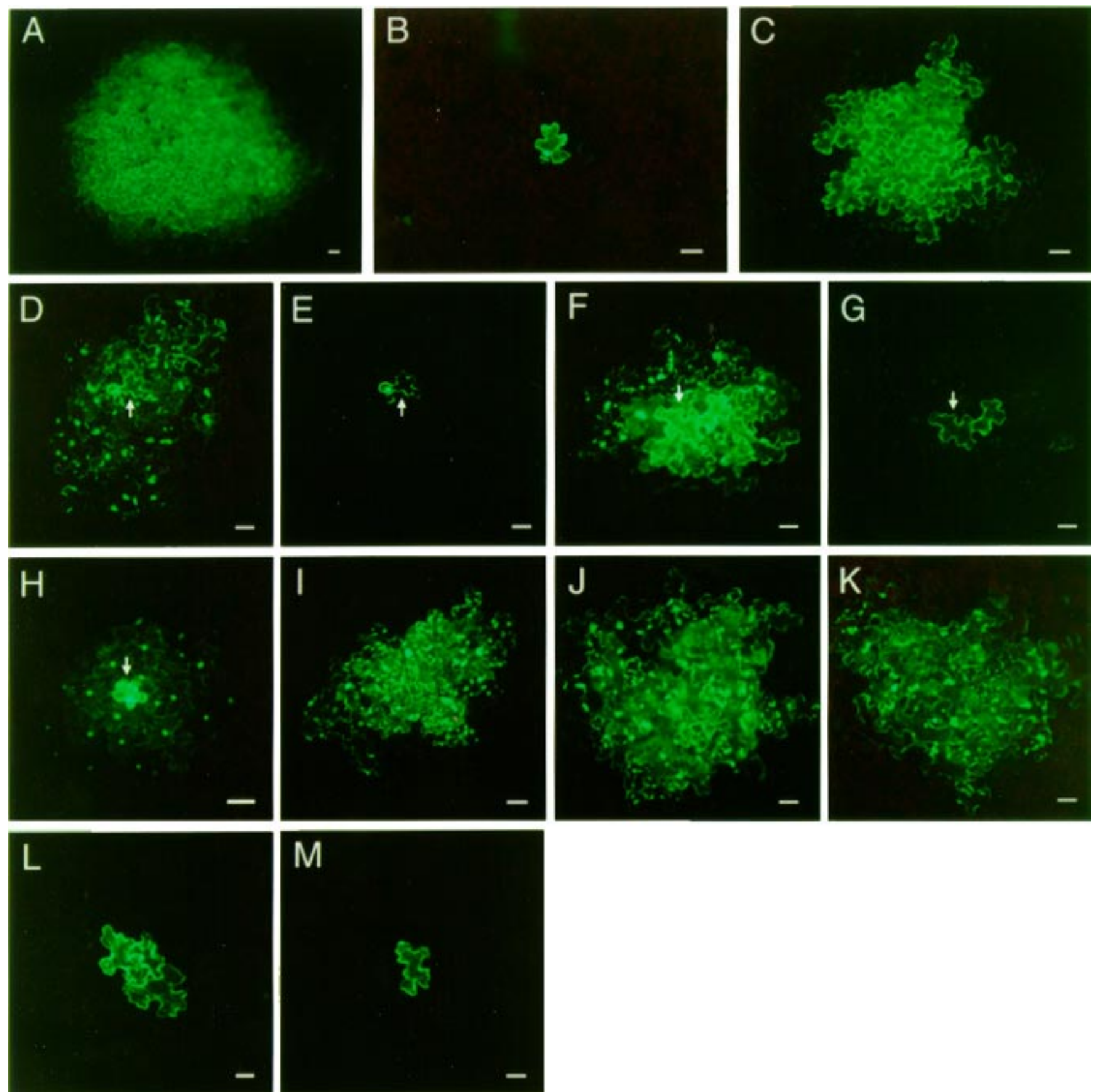

Fig. 2. Transcomplementation of movement-defective Tomato mosaic virus (ToMV). Images were taken under blue light ( $\mathbf{A}$ to $\mathbf{D}$, F, and $\mathbf{H}$ to $\mathbf{M})$ or $\mathrm{UV}$ irradiation (E and $\mathbf{G}$ ) at $48 \mathrm{~h}$ postbombardment. A, Bombardment of piL.G3 encoding the wild-type movement protein (MP) and a green fluorescent protein variant (G3GFP), resulting in multicellular infection. B, Bombardment of piL.G3(SF3) with a frameshift mutation in the MP gene, resulting in single-cell infection. C, Cobombardment of piL.G3(SF3) and p35LM with the 35S-ToMV MP gene, forming a cluster of GFP-positive cells. D to G, Cobombardment of piL.erG3(SF3), a movement-defective construct encoding erG3GFP, together with p35LM and pBINm encoding NmGFP (D and E) or with p35LM and pBImGFP5ER encoding mGFP5ER (F and G). Initially infected (bombarded) cells were visualized by UV irradiation in $\mathbf{E}$ and $\mathbf{G}$, and marked by arrows in $\mathbf{D}$ to G. H, Cobombardment of piL.Nm(SF3), a movement-defective construct encoding NmGFP, together with p35LM and pBIerG3 encoding erG3GFP. Infected cells exhibit nuclear fluorescence derived from virally expressed NmGFP. Arrow = bombarded cell with strong fluorescence of erG3GFP. I to M, Typical patterns obtained when piL.erG3(SF3) was cobombarded with another plasmid expressing ToMV MP(I), Cg-TMV MP (J), SHMV MP (K), CGMMV MP $(\mathbf{L})$, or CMV 3a MP (M). Bars $=50 \mu \mathrm{m}$. 
Transcomplementation of movement-defective ToMV by transiently expressed tobamoviral MP genes.

When an MP-defective construct was bombarded with another plasmid, p35LM, from which ToMV MP was expressed under the control of the $35 \mathrm{~S}$ promoter (Fig. 1B), GFP-positive cells formed clusters (Fig. 2C). This suggests that the defect in cell-to-cell movement was complemented in trans by the cobombarded MP gene. Because the trafficking of GFP itself might be potentiated in the presence of MP, we assessed this possibility prior to further analysis.

G3GFP, initially used as a reporter, is a high-fluorescent GFP variant localized in the nucleus and cytosol (Table 1 and Fig. 3A). When G3GFP alone was expressed, the bombarded sites formed halos at a frequency of approximately 5 and $40 \%$ at 24 and $48 \mathrm{~h}$ postbombardment, respectively, in which one to three cells adjoining a bombarded cell had weak GFP signals (Fig. 3B). When coexpressed with ToMV MP, 50\% or more of the GFP-positive sites formed halos at $24 \mathrm{~h}$ postbombardment (Fig. 3C). Such enhancement of G3GFP diffusion was not observed with an unrelated protein, GAL4-VP16 (data not shown). Although these observations were consistent with a recent report showing the cell-to-cell trafficking of a soluble GFP (Oparka et al. 1999) as well as with the generally accepted activity of MP to promote the cell-to-cell trafficking of macromolecules through plasmodesmata (Waigmann et al. 1994; Wolf et al. 1989; Wolf et al. 1991), the results also indicated that when an active MP is produced, the cytosolic type of GFP variants such as G3GFP can no longer be used as appropriate reporters for explicitly identifying the infected cells.

As an alternative, we examined an ER-localized GFP, erG3GFP (Table 1), which contained the ER-targeting signal at the $\mathrm{N}$ terminus and the ER retention signal at the $\mathrm{C}$ terminus (Fig. 1B). When erG3GFP was expressed singly or together with MP (Fig. 3E and F), green fluorescence was restricted to the bombarded cells and, therefore, erG3GFP was not transported by the ToMV MP. It should be noted that intracellular localization pattern of erG3GFP was apparently modulated in the presence of MP without viral multiplication

Table 2. Multicellular spread of movement-defective Tomato mosaic virus (ToMV) by the cobombarded ToMV movement protein (MP) gene

\begin{tabular}{lccc}
\hline & \multicolumn{2}{c}{ Cell-cell boundaries $^{\mathbf{b}}$} & \multirow{2}{*}{ Total number of infection sites examined } \\
\cline { 2 - 3 } Bombarded plasmids $^{\mathbf{a}}$ & Single & Two or more & $62(100)$ \\
piL.erG3(SF3) + p35LM + pBINm & $8(13)$ & $54(87)$ & $77(82)$ \\
piL.erG3(SF3) + p35LM + pBImGFP5ER & $17(18)$ & $30(88)$ & $34(100)$ \\
piL.Nm(SF3) + p35LM + pBIerG3 & $4(12)$ & $34(100)$ \\
\hline
\end{tabular}

${ }^{a}$ In each combination, the three plasmids, in order, produced movement-defective ToMV (piL.erG3[SF3] and piL.N[SF3]), ToMV MP (p35LM), and a GFP derivative absolutely (pBImGFP5ER and pBIerG3) or mainly (pBINm) localized in the bombarded cells.

${ }^{\mathrm{b}}$ Data are presented as the number of infection sites in which the movement-defective virus spread across the indicated number of cell-cell boundaries at $48 \mathrm{~h}$ postbombardment. Frequency $(\%)$ is shown in parentheses. Single-cell infection was not detected.
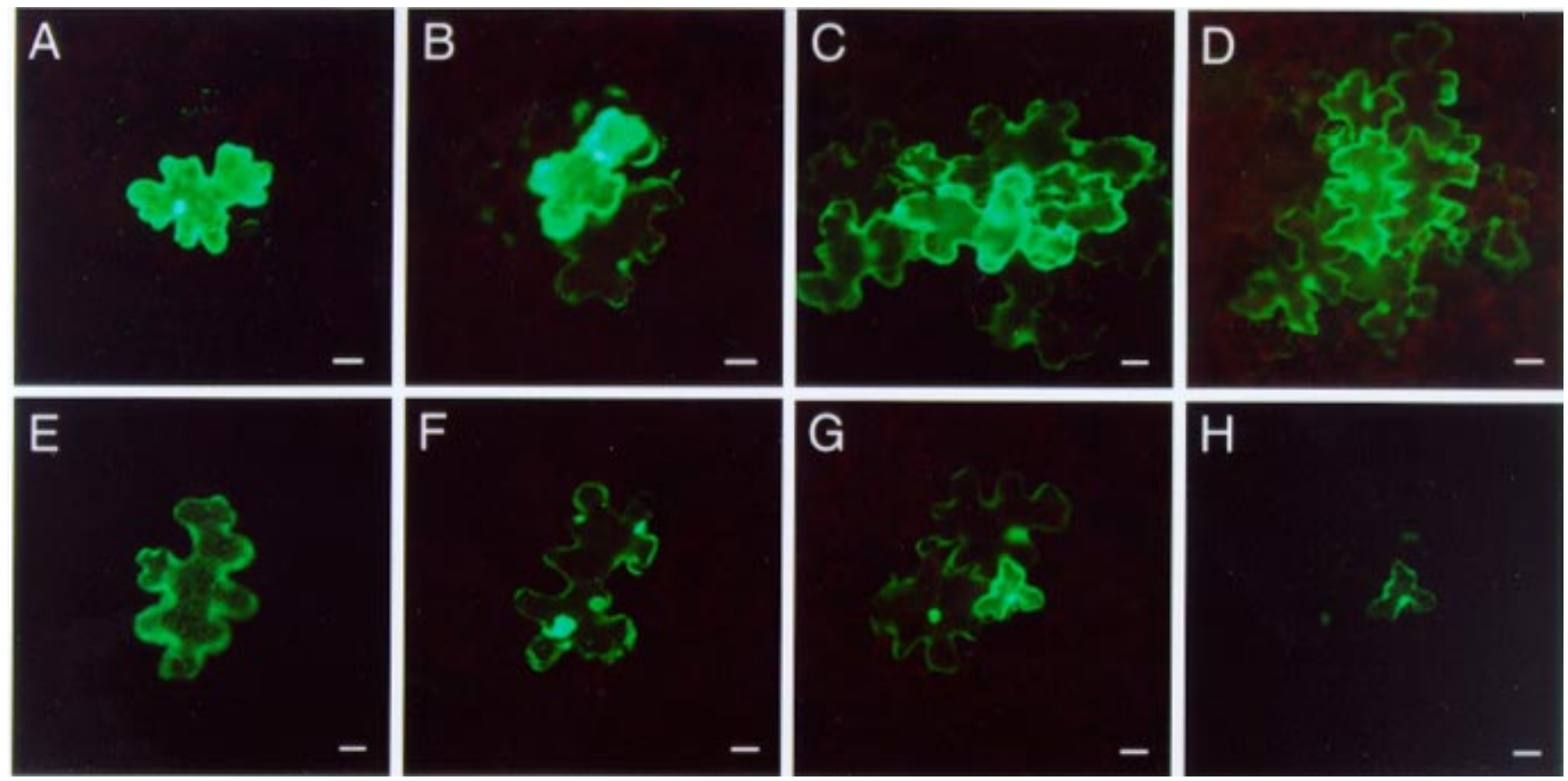

Fig. 3. Distribution patterns of green fluorescent protein (GFP) variants in the presence or absence of MP. Images were taken under blue light (A to G) or UV irradiation $(\mathbf{H})$ at $24 \mathrm{~h}$ postbombardment. A and B, Bombardment of pBIG3 with the 35S-G3GFP gene. G3GFP fluorescence was restricted to the bombarded cell in A or formed a halo in B. C and D, Frequently observed patterns obtained when G3GFP was coexpressed with ToMV MP (p35LM) (C) or CMV 3a MP (p35YM) (D). E and F, Expression of endoplasmic reticulum (ER)-localized erG3GFP (pBIerG3) alone (E) or with ToMV MP (p35LM) (F). erG3GFP was not traffickable. Note that the ER was distorted in the presence of the MP (F). G and H, Coexpression of NmGFP (pBINm) and ToMV MP (p35LM). NmGFP was detected in cells adjoining the bombarded cell (G), but their fluorescence intensity was weak under UV irradiation $(\mathbf{H})$. Bars $=25 \mu \mathrm{m}$. 
(compare Fig. 3E and F), confirming that TMV MP interacts with the ER (Reichel and Beachy 1998).

The activity of the MP gene in transcomplementing the movement-defective phenotype was reexamined by piL.erG3(SF3) encoding erG3GFP in place of the CP (Fig. 1A). In this experiment, the inoculum included a third plasmid expressing NmGFP or mGFP5ER (Fig. 1B and Table 1) to identify the primary infected cells under UV irradiation. mGFP5ER (Haseloff and Siemering 1998) is localized in the ER and is as nontraffickable as erG3GFP. NmGFP had a nuclear localization signal (NLS) at the $\mathrm{N}$ terminus to localize the fluorescence mainly in the nuclei. Because of its small size, NmGFP was not strictly localized in the nucleus and consequently trafficked between cells (Fig. 3G). The fluorescence intensity of the surrounding cells was low, however, particularly under UV irradiation (Fig. $3 \mathrm{H}$ ).

The movement-defective phenotype of the mutant derived from piL.erG3(SF3) was apparently complemented when ToMV MP was coexpressed from p35LM (Fig. 2D to G). Most infection sites contained as many as eight to twenty GFP-positive cells at $48 \mathrm{~h}$ postbombardment. Importantly, the distribution pattern of the virally expressed erG3GFP indicated that in 80 to $90 \%$ of the infection foci, the movementdefective virus spread from the initially infected cells across two or more cell boundaries (Table 2). The results clearly demonstrated that the ToMV MP gene exerts its effects not only in the initially infected cells but also in their surrounding cells.

Essentially the same result was obtained when piL.Nm(SF3), p35LM, and pBIerG3 were cobombarded (Table 2). piL.Nm(SF3) is a movement-defective construct encoding nuclear-localized NmGFP (Fig. 1A), and its strong nuclear fluorescence made it easier to count the number of infected cells (Fig. 2H). In this experiment, the bombarded cells were identified by strong cytoplasmic signals of the ERlocalized erG3GFP derived from pBIerG3 (Figs. 1B and 2H). The distribution pattern of the GFP signals also demonstrated that the mutant virus spread across more than one cell boundary from the bombarded cells.

To delineate at the specificity of transcomplementation, we examined the activity of other tobamoviral MPs derived from TMV, Cg-TMV, Sunn hemp mosaic virus (SHMV), Cucumber green mottle mosaic virus (CGMMV), and Ob-TMV, which were closely or distantly related. For instance, the identity to the ToMV MP at the amino acid sequence level varies from $20 \%$ for SHMV MP to $77 \%$ for TMV MP (Aguilar et al. 1996). When coexpressed with G3GFP, all of these MPs promoted halo formation at similar frequencies (50 to $60 \%$ of the bombarded sites at $24 \mathrm{~h}$ postbombardment), confirming their expression in the bombarded cells and their modulation of the plasmodesmal permeability (data not shown). Cobombardment experiments with piL.erG3(SF3) showed that all of the cells had the ability to transcomplement the movementdefective phenotype (Fig. 2I to L). The efficiency of complementation mediated by CGMMV MP as evaluated by the number of infected cells in each infection site, however, was rather low (Fig. 2L).

We also examined the Cucumber mosaic virus (CMV) 3a MP, which, like TMV MP, is known to be localized in plasmodesmata, binds to single-strand RNA, increases plasmodesmal permeability, and moves from cell to cell (Ding
1998; Lazarowitz and Beachy 1999). Expression of the active 3a MP in the bombarded cells was confirmed by halo formation when coexpressed with the cytosolic G3GFP (Fig. 3D). The CMV 3a MP, however, was unable to complement the defective movement of the ToMV mutant (Fig. 2M), which is consistent with the observation that movement-defective TMV failed to spread in transgenic tobacco expressing the 3a MP (Kaplan et al. 1995).

\section{Facilitation of the intercellular distribution of MP-enhanced green fluorescent protein variant (EGFP) by virus infection.}

The fact that the bombarded MP genes functioned beyond multiple cell-to-cell boundaries implied the presence of MP outside the initially infected cells. For confirmation, we examined the intracellular and intercellular distribution patterns of MP in infected and noninfected sites by means of the fluorescence of a fusion (MP-EGFP) between ToMV MP and an enhanced GFP variant, EGFP (Table 1).

When p35LME harboring the 35S-MP-EGFP gene (Fig. 1B) was bombarded, most of the fluorescence was inside the bombarded cells, accumulating into a number of irregularly shaped aggregates that were sometimes associated with filamentous structures (Fig. 4A). Similar localization patterns have been reported for either virally or singly expressed MPGFPs (Heinlein et al. 1998; Kawakami and Watanabe 1997; Más and Beachy 1999; Reichel et al. 1999). In approximately $80 \%$ of the fluorescent sites, MP-EGFP was restricted only to the bombarded cells (Table 3). In these sites, tiny fluorescent spots in the cell wall, representing plasmodesmal association of MP (Oparka et al. 1997), were rarely found. In the rest of the sites (approximately 20\%), a small amount of MP-EGFP was detected as tiny fluorescent spots in the cell wall surrounding the bombarded cells and one to three adjoining cells at portions not directly connecting to the bombarded cells (Fig. 4B). An increasing number of tiny fluorescent spots was usually accompanied with a decreasing number of intracellular aggregates. These observations suggest that MP-EGFP likely trafficked from cell to cell, although very inefficiently. The MP-EGFP mRNA did not seem to be transported because cytoplasmic aggregates were never found in the neighboring cells.

To reveal the effect of viral infection on the distribution pattern of MP-EGFP, we used piL.Nm(SF3) (Fig. 1) as a movement-defective construct (Fig. 4C to F). Infected cells were identified under UV irradiation as having strongly fluorescing nuclei derived from the virally expressed NmGFP (Table 1). The fluorescence of NmGFP remaining in the cytosol did not interfere with weak signals (fluorescent spots) of MP-EGFP in the cell wall. At $15 \mathrm{~h}$ postbombardment, when MP-EGFP had already accumulated to form aggregates, NmGFP signals were barely found or were too weak to give evidence of infection. At $24 \mathrm{~h}$ postbombardment, the infection was usually up to the immediate neighbors. At or near this stage of infection, MP-EGFP was detected in the cell wall surrounding the bombarded cells as well as between the adjoining (secondary infected) cells (Fig. 4C and D), whereas the cytoplasmic aggregates were diminished in number and size or were undetectable.

At $40 \mathrm{~h}$ postbombardment, infection had reached nearly to maximum size and each infection site contained a cluster of 
infected cells (Fig. 4E and F), indicating that the 35S-MPEGFP gene transcomplemented the movement-defective phenotype. The activity of MP-EGFP (or the final size of infection reflecting the number of cell-to-cell boundaries trafficked) appeared to be slightly lower than that of the authentic MP, and the MP-EGFP signals were detected mainly in the cell wall as punctate spots (Fig. 4E). Remarkably, as many as approximately $90 \%$ of the MP-EGFP-positive foci exhibited multicellular distribution of MP-EGFP (Table 3). It is notable that the MP-EGFP signals in the cell wall were typically found between already infected (NmGFP-positive) cells but rarely between an infected and a detectably uninfected (NmGFP-negative) cell. The bombarded cell sometimes contained cytoplasmic aggregates of MP-EGFP, although their size and number were much smaller than those observed in noninfected cells.

The MP-EGFP fluorescence became rather weak at $40 \mathrm{~h}$ postbombardment. In $25 \%$ of the infection foci, the fluorescence could not be detected even though the virus had spread across more than one cell boundary (Table 3). Finding MPEGFP signals was often difficult at $48 \mathrm{~h}$ postbombardment, whereas large and highly fluorescent intracellular aggregates (Fig. 4A) were still clearly visible in NmGFP-negative cells. It is assumed that replicase proteins were expressed in many, if not all, of these noninfected sites. Actually, the same distribution patterns were obtained when the 35S-MP-EGFP gene was cobombarded with a replication-defective construct lacking the $3^{\prime}$ noncoding region of ToMV (data not shown). Therefore, expression of the replicase gene alone (at least under the control of the $35 \mathrm{~S}$ promoter) appears to be insufficient for modulating the subcellular localization pattern of MP-EGFP. In addition, the cell-to-cell trafficking of MP-EGFP in noninfected sites occurred at a similar level and at the same frequency $(22 \%)$, irrespective of whether the inoculum included the infectious construct (Table 3). Taken together, it is likely that ToMV infection (or the presence of a replicating virus) markedly affects the intracellular and intercellular distribution patterns of the MP-EGFP.

\section{DISCUSSION}

Microinjection studies have shown that bacterially expressed TMV MP can move from cell to cell (Waigmann and Zambryski 1995; Waigmann et al. 1994). The localization of MP produced during infection and analysis of the plasmodesmal gating in expanding infection sites, however, suggested that the virally expressed MP is not transported to as yet unin- fected distant cells (Oparka et al. 1997; Padgett et al. 1996). Further, Storms et al. (1998) revealed that the increase in the plasmodesmal SEL observed in MP-expressing tobacco tissues was a method-dependent phenomenon. Thus, it remains unclear if tobamoviral MP traffics multicellularly in infected sites or how far the MP in a given cell exerts its effects from the MP-expressing cell.

In this work we employed transcomplementation experiments to clarify this problem. Results clearly showed that in $N$. benthamiana the transiently expressed MP gene enables movement-defective ToMV to move from the bombarded cells to their neighbors through two or more cell boundaries. Accompanied by the multicellular spread of the movementdefective virus, the MP (as evaluated by the fluorescence of MP-EGFP) was intercellularly distributed and subsequently localized in plasmodesmata. In contrast, in noninfected sites the cell-to-cell trafficking of MP-EGFP occurred very infrequently. Taken together, it is likely that the MP, concurrently with the viral genome, moved from the initially infected cells to their neighbors as a component of vRNP or a free protein. In the neighboring cells, the MP could be reutilized to move newly replicated viral RNA further away from the cells. Some vRNP (or genomic RNA) might be trafficked through more than one plasmodesma without entering the replication cycle. A possibility still remains that the MP mRNA also might be transported with the genomic RNA in the infected sites.

It is noteworthy that in noninfected cells, the MP-EGFP predominantly accumulated into highly fluorescent intracellular aggregates. Similar intracellular aggregates have been reported for virally expressed MP or MP-GFP fusions in tobacco protoplasts infected with TMV, ToMV, or Ob-TMV (Heinlein et al. 1998; Kawakami and Watanabe 1997; Más and Beachy 1999; Meshi et al. 1992) and in leaf cells infected with TMV or Ob-TMV (Heinlein et al. 1998; Padgett et al. 1996). In the latter case, the cytoplasmic aggregates of MPGFP were found inward from the leading edge of expanding infection sites, whereas in the cells located at the front edge, fluorescent signals of MP-GFP were primarily found in plasmodesmata. Collectively, the cells where MP(-GFP) aggregated were noninfected cells lacking a replicating virus, protoplasts from which a virus cannot move, and infected leaf cells from which active movement may have finished. Therefore, the presence of the cytoplasmic aggregates is not correlated with active cell-to-cell movement of tobamoviruses.

In animal cells, it is known that when misfolded protein is produced at a level exceeding the capacity of proteasomes, socalled aggresomes (proteasome-enriched large aggregates)

Table 3. Effect of infection on intercellular distribution of movement protein-enhanced green fluorescent protein variant (MP-EGFP)

\begin{tabular}{|c|c|c|c|c|c|}
\hline \multirow[b]{3}{*}{ Bombarded plasmid ${ }^{\mathrm{a}}$} & \multicolumn{5}{|c|}{ Distribution of MP-EGFP ${ }^{b}$} \\
\hline & \multicolumn{2}{|c|}{ In uninfected sites } & \multicolumn{3}{|c|}{ In infected sites ${ }^{\mathrm{c}}$} \\
\hline & Single & Two or more $(\%)$ & Not detected & Single & Two or more (\%) \\
\hline p35LME & 206 & $58(22)$ & - & - & - \\
\hline p35LME + piL.Nm(SF3) & 292 & $82(22)$ & 25 & 8 & $71(90)$ \\
\hline \multicolumn{6}{|c|}{$\begin{array}{l}\text { a p35LME encodes the 35S-Tomato mosaic virus (ToMV) MP-EGFP gene; piL.Nm(SF3) is an infectious clone producing a movement-defective ToMV. } \\
\text { b Data are presented as the number of the bombarded sites exhibiting different distribution patterns of MP-EGFP at } 40 \mathrm{~h} \text { postbombardment. Single = MP- } \\
\text { EGFP was detected inside the bombarded cell and/or in the cell wall surrounding the bombarded cell only. Two or more = MP-EGFP was present in the } \\
\text { cell wall not adjoining the bombarded cell (i.e., between two nonbombarded cells). Not detected = MP-EGFP signals were not detected, even though } \\
\text { multiple cells were infected. The percentage of two or more in the total number of MP-EGFP-positive sites are shown in parentheses. } \\
\text { c Infected cells were identified by strongly fluorescing nuclei having virally expressed NmGFP under UV irradiation. All of the infected sites identified } \\
\text { contained a cluster of infected cells. }\end{array}$} \\
\hline
\end{tabular}


develop around the microtubule organization center with the aid of the cytoskeletal network (García-Mata et al. 1999; Johnston et al. 1998). Although the corresponding structures have not been revealed in plant cells, it is possible that the aggregates of MP(-GFP) are likewise formed as a result of the host cell response to sequester problematic proteins, as briefly argued by Más and Beachy (1999). Proteasome-mediated degradation of MP expressed in protoplasts (Reichel and Beachy 2000) and the association of MP with microtubules at relatively later stages of infection (Heinlein et al. 1995; McLean et al. 1995; Padgett et al. 1996) appear to be consistent with the aggresome hypothesis. Thus, the cytoplasmic aggregates may not necessarily represent the potentially active MP.
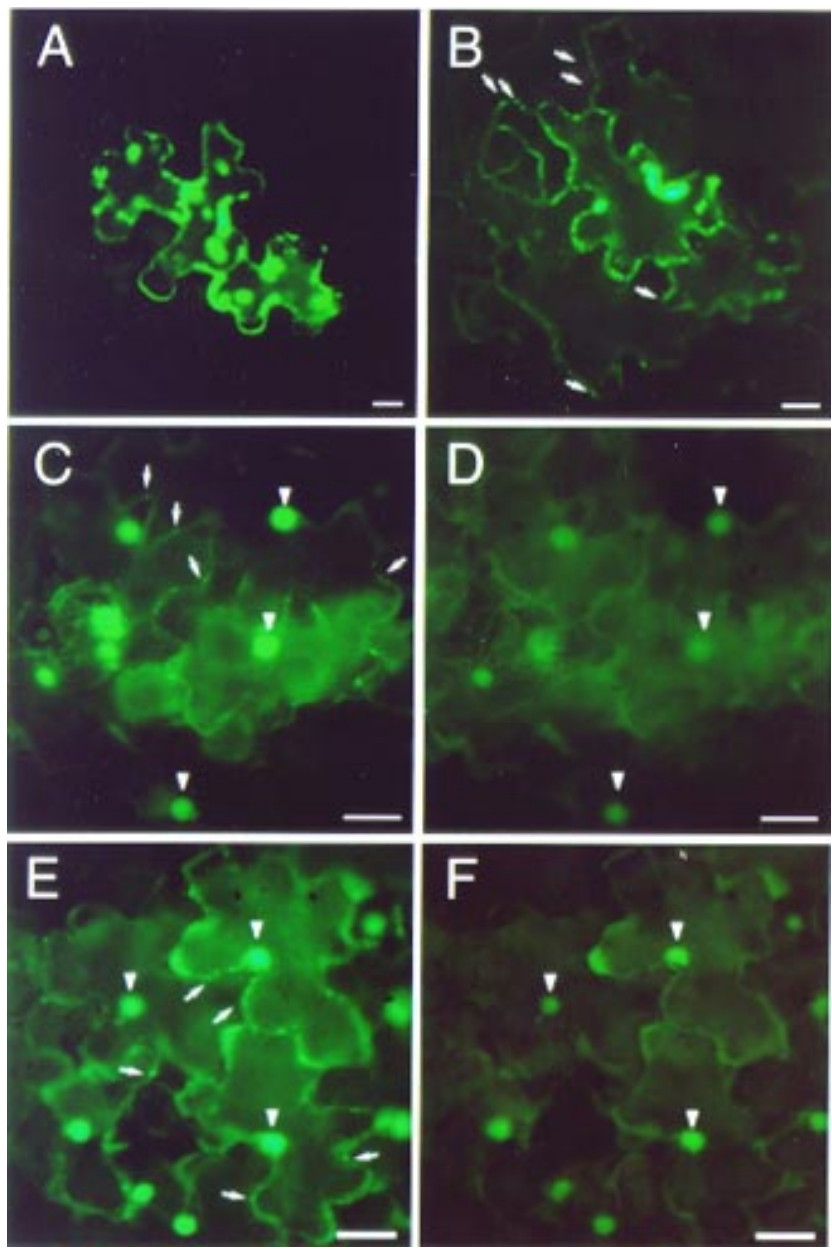

Fig. 4. Different distribution patterns of movement protein (MP)enhanced green fluorescent protein variant (EGFP) in infected and noninfected sites. Images were taken under blue light (A to $\mathbf{C}$ and $\mathbf{E}$ ) or UV irradiation (D and $\mathbf{F})$ at $40(\mathbf{A}, \mathbf{B}, \mathbf{E}$, and $\mathbf{F})$ or $24 \mathrm{~h}$ postbombardment (C and D). A and B, Bombardment of p35LME harboring the Tomato mosaic virus (ToMV) MP-EGFP fusion gene under the control of the $35 \mathrm{~S}$ promoter. MP-EGFP accumulates into multiple aggregates in the bombarded cell (A) or infrequently traffics from the bombarded cell to the neighboring cells (B). Small arrows highlight tiny fluorescent spots in the cell wall, representing plasmodesmal localization of MP-EGFP. C to $\mathbf{F}$, Cobombardment of p35LME with piL.Nm(SF3), an infectious clone producing a movement-defective ToMV. Nuclear-localized NmGFP signals (arrowheads in $\mathbf{D}$ and $\mathbf{F}$ ), indicative of infection, were UV-excitable. Small arrows in $\mathbf{C}$ and $\mathbf{E}=$ plasmodesma-localized MPEGFP signals. Bars $=25 \mu \mathrm{m}$.
As infection proceeded, the amount of MP-EGFP became very low or below the limit of detection. For example, approximately $24 \mathrm{~h}$ after bombardment, most signals of MPEGFP were found in plasmodesmata, whereas only a small fraction was present in the intracellular aggregates. Assuming that the aggregates contain active MP, the change of the distribution pattern of MP-EGFP upon infection may be explained by MP-EGFP (already synthesized and accumulated) being efficiently utilized in the bombarded cells and trafficked to their neighbors. Alternatively, an excess amount of MP-EGFP might be destabilized efficiently upon infection. Another possibility that MP-EGFP might be expressed at a low level, specifically in the cells later undergoing infection, seems unlikely considering the following observations: i) GFP derivatives used as reporters to identify the bombarded cells were stably detected in infected and noninfected cells; ii) aggregates of MP-EGFP were detected much earlier than the time when infected cells were recognized by the expression of a virally expressed GFP reporter; and iii) MP-EGFP was stably detected in noninfected cells.

It is evident that there is a remarkable difference in the distribution patterns of MP(-EGFP) between infected and noninfected sites and that the final distribution pattern (multicellular spread and plasmodesmal localization) of MP in infected sites agrees with its cell-to-cell movement function. Apart from the mechanisms, the fact that the behavior of MP is greatly affected by infection suggests that the MP requires some factor specifically induced in the infected cells to fulfill its activity to transport the tobamoviral genome from cell to cell.

It has been shown that TMV MP colocalizes with vRNA and the replicase component, at least at certain stages of infection in tobacco protoplasts or leaf cells (Heinlein et al. 1998; Más and Beachy 1999; Szécsi et al. 1999). Although such colocalization has yet to be observed in leaf cells from which the virus actively moves, their finding suggests that MP is produced in close proximity to the replication machinery in infected leaf cells. Together with our observations that multicellular spread of MP in leaves is greatly enhanced in the presence of a replicating virus and likely coordinates with cell-to-cell movement of the viral genome, it is tempting to examine whether tobamoviral MP interacts, directly or indirectly, with replication machinery components.

\section{MATERIALS AND METHODS}

\section{Construction of infectious ToMV plasmids.}

The CaMV 35S RNA promoter sequence ( -864 to -1 , relative to the transcription initiation site) was polymerase chain reaction (PCR)-amplified from pBI221 (Clontech, Palo Alto, CA, U.S.A.) with a set of oligonucleotide primers designed to have an AatI site between -1 and +1 (Yamaya et al. 1988) and $\mathrm{SacI}$ and $\mathrm{Xba \textrm {I }}$ sites at the ends. The amplified fragment was cloned between the $S a c \mathrm{I}$ and $X b a \mathrm{I}$ sites of pBluescriptSK+ (Stratagene, La Jolla, CA, U.S.A.) to generate pb35S. PCRamplified regions and ligation junctions in all constructions were confirmed by sequencing.

Infectious plasmids (piL series) (Fig. 1A) were made via several intermediates in which the wild-type ToMV cDNA sequence was derived from pLFW3 (Meshi et al. 1986). Briefly, a PCR-amplified ToMV double-stranded cDNA (from nucleotide 1 to 1,229 [SpeI site]) was inserted between the 
AatI and SpeI sites of pb35S to generate p35ToMV5 in which the transcription initiation site corresponded to the $5^{\prime}$ end of ToMV. The SpeI-SmaI fragment of pLFW3, corresponding to the 3' part of the ToMV sequence (from nucleotides 6,099 to the $3^{\prime}$ end) was ligated to the nopaline synthase gene terminator (the blunt-ended SacI-EaeI fragment of pBI221), then inserted between the SpeI and blunted KpnI sites of p35ToMV5 to yield p35L $\Delta \mathrm{N}$. The internal part of the ToMV sequence was inserted into the SpeI site of p35L $\Delta \mathrm{N}$ to generate the full-length construct piLW3. The CP and MP genes were modified on subclones then returned to piLW3 or an appropriate infectious clone. piL.G3 (Fig. 1) had the SacI (blunted)-BstEII fragment of pTLQG3::fus (Kawakami and Watanabe 1997) encoding G3GFP plus a 19-bp linker-derived sequence in place of the ToMV sequence from nucleotide 5,718 (the sixth codon of the CP gene) to 5,799 (BstEII site). G3GFP expressed from piL.G3 had 12 extra amino acids at the $\mathrm{N}$ terminus (MSYSIPISGGGG) prior to the authentic methionine (Met) of GFP, in which the first five amino acids (underlined) were identical to those of the ToMV CP. piL.G3(SF3), as well as other plasmids with (SF3) in their name, had a 1-bp deletion at nucleotide 4,935, resulting in frame-shifting at the tenth codon of the MP gene as in pLQSF3 (Meshi et al. 1987). The sequence from nucleotide 5,703 (corresponding to the initiation codon of the $\mathrm{CP}$ gene) to 6,098 (SpeI site) of piL.G3(SF3) was replaced with a fragment encoding erG3GFP (from the initiation codon to the SacI site of pBIerG3, as described below) to yield piL.erG3(SF3). erG3GFP expressed from piL.erG3(SF3) had no CP-derived amino acids at the $\mathrm{N}$ terminus. To create piL.Nm(SF3), the G3GFP gene of piL.G3(SF3) was replaced with a synthetic double-stranded oligonucleotide encoding the NLS of SV40 large-T antigen and the EcoRI-SacI fragment of pBINmGFP5ER just encoding mGFP5 (Haseloff and Siemering 1998; Haseloff et al. 1997). NmGFP expressed from piL.Nm(SF3) had MSYSIPELPPKKKRKV-EF at the N terminus, followed by mGFP5, in which five CP-derived amino acids are underlined.

\section{Plasmids for expression of MP, GFP, and MP-EGFP.}

Each of the fragments encoding a tobamoviral or cucumoviral MP was PCR-amplified with an appropriate cDNA clone as template and inserted between the $\mathrm{XbaI}$ and $\mathrm{SacI}$ sites of pBI221. The resulting plasmids, p35LM (Fig. 1B), p35CgM, p35OMM, p35CcM, p35WM, p35ObM, and p35YM, contained the MP gene of ToMV (L strain), Cg-TMV, TMV (OM strain), SHMV, CGMMV (W strain), Ob-TMV, and CMV (Y strain), respectively. In all cases, the first ATG from the transcription initiation site corresponded to the authentic initiation codon. The plasmid pBImGFP5ER was constructed by replacing the BamHI-SacI fragment of pBINmGFP5ER for the corresponding fragment of pBI221. The larger BamHI (blunted) $-N s p \mathrm{~V}$ fragment of $\mathrm{pBImGFP5ER}$ was ligated with the $S a c$ I (blunted) $-N s p \mathrm{~V}$ fragment of pTLQG3::fus encoding G3GFP to generate pBIG3 (Fig. 1B). The expressed G3GFP had no extra amino acids at the $\mathrm{N}$ terminus and four additional amino acids (HDEL) derived from mGFP5ER at the $\mathrm{C}$ terminus. To express erG3GFP, pBIerG3 (Fig. 1B) was constructed by replacing an $\mathrm{NcoI}$ fragment of pBIG3 with the corresponding fragment of pBImGFP5ER to introduce the sequence for the N-terminal signal peptide. pBINm (Fig. 1B) was constructed by inserting an AatII (blunted)-SacI fragment of piL.Nm between the BamHI (blunted) and SacI sites of pBI221. To create the MP-EGFP fusion gene, a point mutation was introduced at ToMV nucleotide 5,686 to generate an EcoRI site in a pBluescript-based subclone into which the EGFP gene excised from pEGFPIRESneo (Clontech) was inserted. The resulting MP-EGFP fusion gene was then inserted between the blunted $\mathrm{XbaI}$ and $\mathrm{SacI}$ sites of pBI221 to yield p35LME (Fig. 1B). The MP-EGFP lacked the last five amino acids (DSDSY) of the ToMV MP and instead had the linker-derived 11 amino acids NSVDPRVPVAT before the first Met of EGFP.

\section{Microprojectile bombardment.}

Bombardment was performed with PDS1000 (Bio-Rad Laboratories, Hercules, CA, U.S.A.), essentially according to the manufacturer's instructions. Briefly, mature or nearly expanded leaves ( 8 to $10 \mathrm{~cm}$ in length) of $N$. benthamiana (6 to 8 weeks old) were cut and placed on a Murashige-Skoog agar plate at a target distance of $6 \mathrm{~cm}$. Three $\mathrm{mg}$ of gold particles ( $1 \mu \mathrm{m}$ in diameter) were coated with $5 \mu \mathrm{g}$ of plasmid DNA and divided into six equal parts, each of which was subjected to a shot with a rupture disk of $1,350 \mathrm{lb} / \mathrm{in}^{2}$. In cobombardment experiments, equal amounts of the respective plasmids ( $5 \mu \mathrm{g}$ total) were mixed before coating the gold particles. A single leaf was bombarded twice, then incubated at $26^{\circ} \mathrm{C}$ in the dark. At appropriate times after bombardment, leaves were cut into pieces and GFP signals were observed under an Axioskop epifluorescent microscope (Carl Zeiss Inc., Thornwood, NY, U.S.A.). Images were obtained by a color-chilled 3CCD camera, C5810 (Hamamatsu Photonics, Hamamatsu, Japan). Filter set 41014 (Chroma Technologies, Brattleboro, VT, U.S.A.) or No. 10 (Carl Zeiss Inc.) was used to observe GFP variants under blue-light irradiation; filter set 31022 (Chroma Technologies) was used under UV irradiation.

\section{ACKNOWLEDGMENTS}

We thank J. Haseloff, S. Kawakami, W. Watanabe, and M. Ishikawa for plasmids, and M. Iwabuchi and Y. Okada for encouragement. This work was supported in part by Grants-in-Aid for Scientific Research from the Ministry of Education, Science, Sports and Culture of Japan.

\section{LITERATURE CITED}

Aguilar, I., Sánchez, F., Martín, A. M., Martínez-Herrera, D., and Ponz, F. 1996. Nucleotide sequence of Chinese rape mosaic virus (oilseed rape mosaic virus), a crucifer tobamovirus infectious on Arabidopsis thaliana. Plant Mol. Biol. 30:191-197.

Atabekov, J. G., and Taliansky, M. E. 1990. Expression of a plant viruscoded transport function by different viral genomes. Adv. Virus Res. 38:201-248.

Atkins, D., Hull, R., Wells, B., Roberts, K., Moore, P., and Beachy, R. N. 1991. The tobacco mosaic virus $30 \mathrm{~K}$ movement protein in transgenic tobacco plants is localized to plasmodesmata. J. Gen. Virol. 72:209-211.

Carrington, J. C., Kasschau, K. D., Mahajan, S. K., and Schaad, M. C. 1996. Cell-to-cell and long-distance transport of viruses in plants. Plant Cell 8:1669-1681.

Citovsky, V. 1999. Tobacco mosaic virus: A pioneer of cell-to-cell movement. Phil. Trans. R. Soc. Lond. Biol. Sci. 354:637-643.

Citovsky, V., Knorr, D., Schuster, G., and Zambryski, P. 1990. The P30 movement protein of tobacco mosaic virus is a single-strand nucleic acid binding protein. Cell 60:637-647.

Citovsky, V., Wong, M. L., Shaw, A. L., Prasad, B. V. V., and Zambryski, P. 1992. Visualization and characterization of tobacco 
mosaic virus movement protein binding to single-stranded nucleic acids. Plant Cell 4:397-411.

Deom, C. M., Oliver, M. J., and Beachy, R. N. 1987. The 30-kilodalton gene product of tobacco mosaic virus potentiates virus movement. Science 237:389-394.

Deom, C. M., Lapidot, M., and Beachy, R. N. 1992. Plant virus movement proteins. Cell 69:221-224

Ding, B. 1998. Intercellular protein trafficking through plasmodesmata. Plant Mol. Biol. 38:279-310.

Ding, B., Haudenshield, J. S., Hull, R. J., Wolf, S., Beachy, R. N., and Lucas, W. L. 1992. Secondary plasmodesmata are specific sites of localization of the tobacco mosaic virus movement protein in transgenic tobacco plants. Plant Cell 4:915-928.

García-Mata, R., Bebök, Z., Sorscher, E. J., and Sztul, E. S. 1999. Characterization and dynamics of aggresome formation by a cytosolic GFP-chimera. J. Cell Biol. 146:1239-1254.

Haseloff, J., and Siemering, K. R. 1998. The uses of green fluorescent protein in plants. Pages 191-220 in: Green Fluorescent Protein: Properties, Applications, and Protocols. M. Chalfie and S. Kain, eds. Wiley-Liss, New York.

Haseloff, J., Siemering, K. R., Prasher, D. C., and Hodge, S. 1997. Removal of a cryptic intron and subcellular localization of green fluorescent protein are required to mark transgenic Arabidopsis plants brightly. Proc. Natl. Acad. Sci. USA 94:2122-2127.

Heinlein, M., Epel, B. L., Padgett, H. S., and Beachy, R. N. 1995. Interaction of tobamovirus movement proteins with the plant cytoskeleton. Science 270:1983-1985.

Heinlein, M., Padgett, H. S., Gens, J. S., Pickard, B. G., Casper, S. J., Epel, B. L., and Beachy, R. N. 1998. Changing patterns of localization of the tobacco mosaic virus movement protein and replicase to the endoplasmic reticulum and microtubules during infection. Plant Cell 10:1107-1120.

Johnston, J. A., Ward, C. L., and Kopito, R. R. 1998. Aggresomes: A cellular response to misfolded proteins. J. Cell Biol. 143:18831898.

Kaplan, I. B., Shintaku, M. H., Li, Q., Zhang, L., Marsh, L. E., and Palukaitis, P. 1995. Complementation of virus movement in transgenic tobacco expressing the cucumber mosaic virus 3a gene. Virology 209:188-199.

Kawakami, S., and Watanabe, Y. 1997. Use of green fluorescent protein as a molecular marker tag of protein movement in vivo. Plant Biotech. 14:127-130.

Kawakami, S., Padgett, H., Hosokawa, D., Okada, Y., Beachy, R. N., and Watanabe, Y. 1999. Phosphorylation and/or presence of serine 37 in the movement protein of tomato mosaic tobamovirus is essential for intracellular localization and stability in vivo. J. Virol. 73:6831-6840.

Lazarowitz, S. G., and Beachy, R. N. 1999. Viral movement proteins as probes for intracellular and intercellular trafficking in plants. Plant Cell 11:535-548.

Lucas, W. J., Ding, B., and Van der Schoot, C. 1993. Plasmodesmata and the supracellular nature of plants. New Phytol. 125:435-476.

Más, P., and Beachy, R. N. 1999. Replication of tobacco mosaic virus on endoplasmic reticulum and role of the cytoskeleton and virus movement protein in intracellular distribution of viral RNA. J. Cell Biol. 147:945-958

Maule, A. J. 1991. Virus movement in infected plants. Crit. Rev. Plant Sci. 9:457-473.

McLean, B. G., Zupan, J., and Zambryski, P. C. 1995. Tobacco mosaic virus movement protein associates with the cytoskeleton in tobacco cells. Plant Cell 7:2101-2114.

Meshi, T., Ishikawa, M., Motoyoshi, F., Semba, K., and Okada, Y. 1986. In vitro transcription of infectious RNAs from full-length cDNAs of tobacco mosaic virus. Proc. Natl. Acad. Sci. USA 83:5043-5047.

Meshi, T., Watanabe, Y., Saito, T., Sugimoto, A., Maeda, T., and Okada, Y. 1987. Function of the 30kd protein of tobacco mosaic virus: Involvement in cell-to-cell movement and dispensability for replication. EMBO J. 6:2557-2563.

Meshi, T., Hosokawa, D., Kawagishi, M., Watanabe, Y., and Okada, Y. 1992. Reinvestigation of intracellular localization of the $30 \mathrm{~K}$ protein in tobacco protoplasts infected with tobacco mosaic virus RNA. Virology 187:809-813.

Morozov, S. Y., Fedorkin, O. N., Jüttner, G., Schiemann, J., Baulcombe, D. C., and Atabekov, J. G. 1997. Complementation of a potato virus X mutant mediated by bombardment of plant tissues with cloned viral movement protein genes. J. Gen. Virol. 78:2077-2083.

Oparka, K. J., Prior, D. A. M., Santa Cruz, S., Padgett, H. S., and Beachy, R. N. 1997. Gating of epidermal plasmodesmata is restricted to the leading edge of expanding infection sites of Tobacco mosaic virus (TMV). Plant J. 12:781-789.

Oparka, K. J., Roberts, A. G., Boevink, P., Santa Cruz, S., Roberts, I., Pradel, K. S., Imlau, A., Kotlizky, G., Sauer, N., and Epel, B. 1999. Simple, but not branched, plasmodesmata allow the nonspecific trafficking of proteins in developing tobacco leaves. Cell 97:743-754.

Padgett, H. S., Epel, B. L., Kahn, T. W., Heinlein, M., Watanabe, Y., and Beachy, R. N. 1996. Distribution of tobamovirus movement protein in infected cells and implications for cell-to-cell spread of infection. Plant J. 10:1079-1088.

Reichel, C., and Beachy, R. N. 1998. Tobacco mosaic virus infection induces severe morphological changes of the endoplasmic reticulum. Proc. Natl. Acad. Sci. USA 95:11169-11174.

Reichel, C., and Beachy, R. N. 2000. Degradation of tobacco mosaic virus movement protein by the $26 \mathrm{~S}$ proteasome. J. Virol. 74:3330-3337.

Reichel, C., Más, P., and Beachy, R. N. 1999. The role of the ER and cytoskeleton in plant viral trafficking. Trends Plant Sci. 4:458-462.

Szécsi, J., Ding, X. S., Lim, C. O., Bendahmane, M., Cho, M. J., Nelson, R. S., and Beachy, R. N. 1999. Development of tobacco mosaic virus infection sites in Nicotiana benthamiana. Mol. Plant-Microbe Interact. 12:143-152.

Storms, M. M. H., Van der Schoot, C., Prins, M., Kormelink, R., Van Lent, J. W. M., and Goldbach, R. W. 1998. A comparison of two methods of microinjection for assessing altered plasmodesmal gating in tissues expressing viral movement proteins. Plant J. 13:131-140.

Takamatsu, N., Ishikawa, M., Meshi, T., and Okada, Y. 1987. Expression of bacterial chloramphenicol acetyltransferase gene in tobacco plants mediated by TMV-RNA. EMBO J. 6:307-311.

Tomenius, K., Clapham, D., and Meshi, T. 1987. Localization by immunogold cytochemistry of the virus-coded $30 \mathrm{~K}$ protein in plasmodesmata of leaves infected with tobacco mosaic virus. Virology 160:363-371.

Waigmann, E., and Zambryski, P. 1995. Tobacco mosaic virus movement protein-mediated protein transport between trichome cells. Plant Cell 7:2069-2079.

Waigmann, E., Lucas, W. J., Citovsky, V., and Zambryski, P. 1994. Direct functional assay for tobacco mosaic virus cell-to-cell movement protein and identification of a domain involved in increasing plasmodesmal permeability. Proc. Natl. Acad. Sci. USA 91:1433-1437.

Wolf, S., Deom, C. M., Beachy, R. N., and Lucas, W. J. 1989. Movement protein of tobacco mosaic virus modifies plasmodesmatal size exclusion limit. Science 246:377-379.

Wolf, S., Deom, C. M., Beachy, R., and Lucas, W. J. 1991. Plasmodesmatal function is probed using transgenic tobacco plants that express a virus movement protein. Plant Cell 3:593-604.

Yamaya, J., Yoshioka, M., Meshi, T., Okada, Y., and Ohno, T. 1988. Expression of tobacco mosaic virus RNA in transgenic plants. Mol. Gen. Genet. 211:520-525. 